\title{
Modeling PTEN overexpression-induced microcephaly in human brain organoids
}

\author{
Navroop Dhaliwal ${ }^{1 \dagger}$, Wendy W.Y. Choi ${ }^{1,2,3 \dagger}$, Julien Muffat ${ }^{1,2,4}$ and Yun $\mathrm{Li}^{1,2^{*}}$ (0)
}

\begin{abstract}
The phosphatase and tensin homolog (PTEN) protein, encoded by the PTEN gene on chromosome 10, is a negative regulator of the phosphoinositide 3-kinase (PI3K) signaling pathway. Loss of PTEN has been linked to an array of human diseases, including neurodevelopmental disorders such as macrocephaly and autism. However, it remains unknown whether increased dosage of PTEN can lead to human disease. A recent human genetics study identifies chromosome 10 microduplication encompassing PTEN in patients with microcephaly. Here we generated a human brain organoid model of increased PTEN dosage. We showed that mild PTEN overexpression led to reduced neural precursor proliferation, premature neuronal differentiation, and the formation of significantly smaller brain organoids. PTEN overexpression resulted in decreased AKT activation, and treatment of wild-type organoids with an AKT inhibitor recapitulated the reduced brain organoid growth phenotypes. Together, our findings provide functional evidence that PTEN is a dosage-sensitive gene that regulates human neurodevelopment, and that increased PTEN dosage in brain organoids results in microcephaly-like phenotypes.
\end{abstract}

Keywords: PTEN, AKT, Brain organoids, Human pluripotent stem cells, Neural precursors, Microcephaly, Neurodevelopmental disorder

Loss of function mutations in the PTEN tumor suppressor gene are implicated in a wide spectrum of human diseases. In the central nervous system, loss of PTEN leads to brain cancers, as well as non-malignant conditions including macrocephaly, autism, and epilepsy [1]. However, the impact of PTEN overexpression on human health remains largely unknown. Partial trisomy of chromosome 10, including distal 10q where PTEN resides, has been found in patients with severe developmental disorders including craniofacial malformations. Recently, a 10q23.31 microduplication has been identified in patients with autosomal dominant primary microcephaly [2]. PTEN is one of the 3 genes duplicated in this region, and is hypothesized to be the causal gene for the

\footnotetext{
*Correspondence: yun.li@sickkids.ca

${ }^{\dagger}$ Navroop Dhaliwal and Wendy W.Y. Choi contributed equally to this work

${ }^{2}$ Department of Molecular Genetics, University of Toronto, 1 King's College Circle, Toronto M5S 1A8, ON, Canada

Full list of author information is available at the end of the article
}

condition. However, the functional impact of PTEN overexpression on human neurodevelopment has not been experimentally examined.

The advent of human pluripotent stem cells (hPSCs) and 3-dimensional brain organoid technologies provides a new avenue to investigate human neurodevelopment in vitro. We and others have previously utilized these tools to model primary microcephaly caused by genetic mutations [3-6] and environmental factors such as the Zika virus [7-9]. To understand the role of PTEN loss of function in regulating human neurodevelopment, we have previously generated PTEN knockout hPSCs [10]. PTEN knockout brain organoids are significantly larger in size, mimicking the macrocephalic conditions seen in patients with PTEN loss of function mutations.

Here, we generated an hPSCs-derived brain organoid model of mild PTEN overexpression (PTEN-OE) to study the effect of increased PTEN dosage on neurodevelopment. Wild-type WIBR3 hPSCs were transduced with lentivirus expressing a PTEN-GFP fusion cDNA [10].

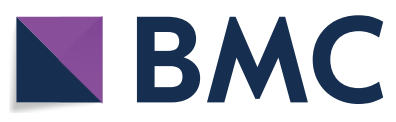

(c) The Author(s) 2021. Open Access This article is licensed under a Creative Commons Attribution 4.0 International License, which permits use, sharing, adaptation, distribution and reproduction in any medium or format, as long as you give appropriate credit to the original author(s) and the source, provide a link to the Creative Commons licence, and indicate if changes were made. The images or other third party material in this article are included in the article's Creative Commons licence, unless indicated otherwise in a credit line to the material. If material is not included in the article's Creative Commons licence and your intended use is not permitted by statutory regulation or exceeds the permitted use, you will need to obtain permission directly from the copyright holder. To view a copy of this licence, visit http://creativecommons.org/licenses/by/4.0/. The Creative Commons Public Domain Dedication waiver (http://creativeco mmons.org/publicdomain/zero/1.0/) applies to the data made available in this article, unless otherwise stated in a credit line to the data. 
GFP-positive subclones were selected (Fig. 1A) and examined for their PTEN expression levels using quantitative RT-PCR. We identified three subclones with mild overexpression of PTEN (Fig. 1B) and further verified their PTEN protein levels using immuno-blotting (Fig. 1C,
D). Three subclones of the same parental WIBR3 hPSCs were used as controls. We next generated forebrain organoids by directed differentiation $[4,10]$. Equal numbers of control and PTEN-OE hPSCs were aggregated to form embryoid bodies of similar size and morphology (Fig. 1F
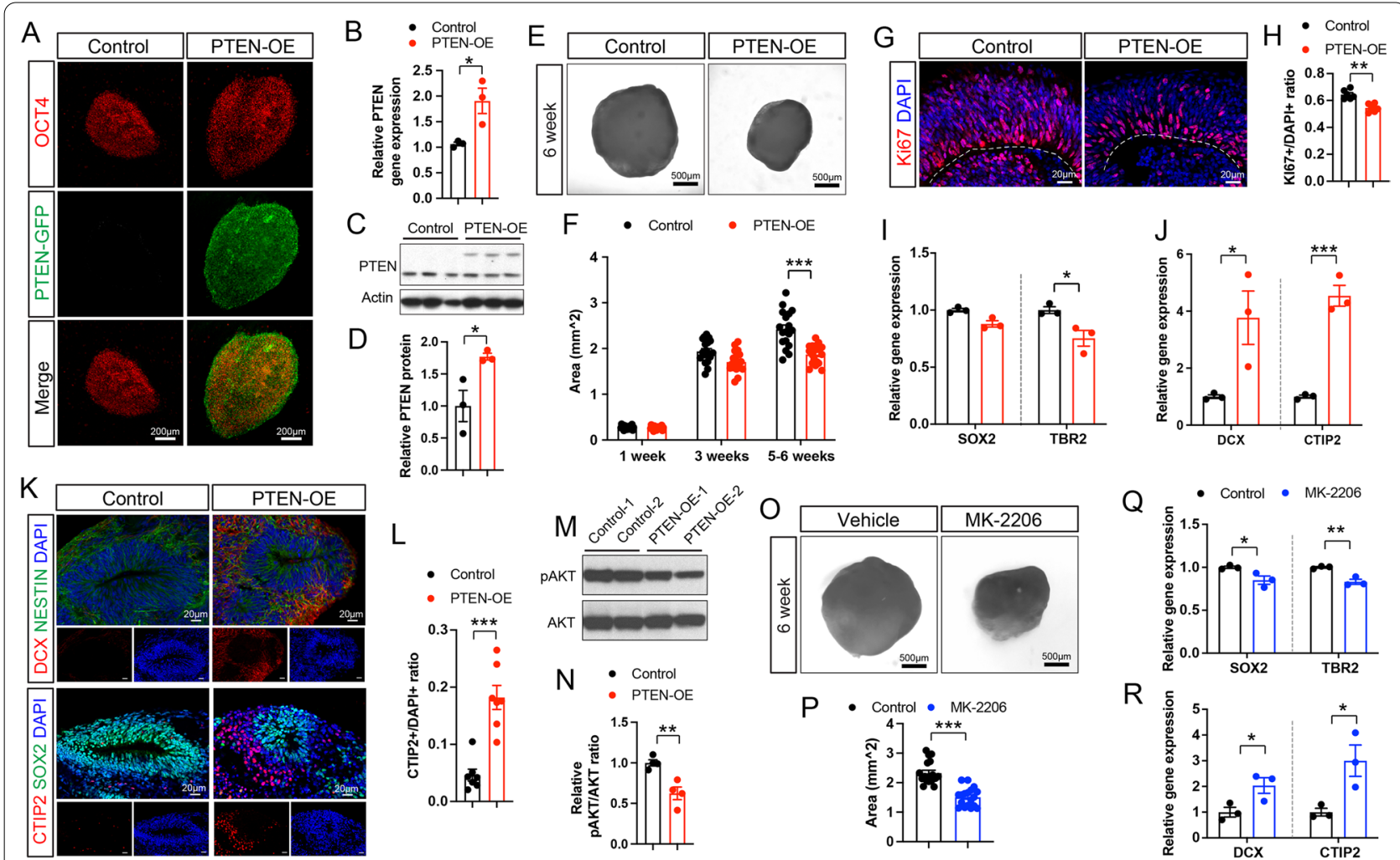

Fig. 1 PTEN-OE brain organoids model human microcephaly. A Immuno-staining of control and PTEN-OE hPSCs for markers of pluripotency (OCT4). Presence of GFP indicates overexpression of PTEN-GFP fusion protein. B Quantitative RT-PCR shows increased expression of PTEN in PTEN-OE hPSCs. Each data point represents one independent hPSC line ( $n=3$ for each group). $\mathbf{C}$ Immuno-blotting analysis showsincreased total PTEN protein level (higher molecular weight band indicates PTEN-GFP fusion protein) in PTEN-OE hPSCs. D Quantification of total PTEN protein levels (endogenous PTEN and PTEN-GFP) in control and PTEN-OE hPSCs, normalized to Actin. Each data point represents one independent hPSC line ( $n=3$ for each group). E Representative images of control and PTEN-OE organoids at 6 weeks. F Quantification of control and PTEN-OE organoid size at around 1 week (day 7-10, 3 weeks (day 21-24), and 5-6 weeks (day 35-42). Each data point represents the area of a single organoid. $\mathrm{N}=18$ for each group at each time point from $3 \mathrm{hPSC}$ lines and 2 independent differentiation experiments. ANOVA revealed significant effects of age $\left(F_{2,102}=669.4, p<0.0001\right)$, genotype $\left(F_{1,102}=34.65, p<0.0001\right)$, and the interaction between the two $\left(F_{2,102}=12.20, p<0.0001\right)$. $\mathbf{G}$ and $\mathbf{H}$ Representative images $(\mathbf{G})$ and quantification $(\mathbf{H})$ of KI67 immuno-staining in 3-week-old control and PTEN-OE brain organoids. White dashed lines indicate the apical edge of the ventricular zone. Each data point represents one hPSC line in an independent differentiation, from the average of measurement from 3 organoids. $\mathrm{N}=6$ for each group from 3 hPSC lines and 2 independent differentiation experiments. I and $\mathbf{J}$ Quantitative RT-PCR analysis of neural precursor markers SOX2, TBR2 (I) and neuronal markers DCX, CTIP2 (J) in 3-week-old control and PTEN-OE brain organoids. Each data point represents one independent hPSC line ( $n=3$ for each group). K Representative images of immuno-staining for DCX and NESTIN (upper panels), CTIP2 and SOX2 (lower panels) in 3-week-old control and PTEN-OE organoids. L Quantification of CTIP2 immuno-staining in 3-week-old control and PTEN-OE brain organoids. Each data point represents one hPSC line in an independent differentiation, from the average of measurement from 3 organoids. $\mathrm{N}=6$ for each group from $3 \mathrm{hPSC}$ lines and 2 independent differentiation experiments. $\mathbf{M}$ Immuno-blotting analysis shows reduced phospho-AKT protein level in 3-week-old PTEN-OE brain organoids generated from hPSC line Control-1, Control-2, PTEN-OE-1, and PTEN-OE-2. N Quantification of immuno-blotting results (Figure 1M and Additional file 1: Figure S1D) shows reduced phospho-AKT to total AKT ratio in 3-week-old PTEN-OE brain organoids. Each data point represents one hPSC line in an independent differentiation. $\mathrm{N}=4$ for each group from $3 \mathrm{hPSC}$ lines and 2 independent experiments. $\mathbf{O}$ Representative images of 6-week-old brain organoids treated with vehicle or AKT inhibitor MK-2206 (100 nM). P Size quantification of vehicle and MK-2206 treated brain organoids at 5-6 weeks. Each data point represents the area of a single organoid. $\mathrm{N}=18$ for each group from $3 \mathrm{hPSC}$ lines and 2 independent differentiation experiments. Q and $\mathbf{R}$ Quantitative RT-PCR analysis of neural precursor markers SOX2, TBR2 (Q) and neuronal markers DCX, CTIP2 (R) in vehicle and MK-2206 treated 6-week-old brain organoids. Each data point represents one independent hPSC line ( $n=3$ for each group). Results are mean $\pm \mathrm{SEM} .{ }^{*} p<0.05,{ }^{* *} p<0.01,{ }^{* * *} p<0.001$ 
and Additional file 1: Figure S1A, B). These embryoid bodies were cultured in neural differentiation medium and embedded in Matrigel droplets to form forebrain organoids. We observed that the growth of PTEN-OE organoids was slower compared to controls, and PTENOE organoids were significantly smaller at 5-6weeks (Fig. 1E, F). PTEN overexpression in embryoid bodies and organoids was confirmed using quantitative RT-PCR (Additional file 1: Figure S1C). To investigate whether the reduced organoid growth was linked to altered cellular proliferation, we next performed immuno-staining for Ki67. Within the ventricular zone where neural precursors reside, fewer Ki67-positive cells were observed in 3-week-old PTEN-OE organoids compared to their isogenic controls (Fig. 1G, H). This reduction coincided with decreased neural precursor markers (SOX2, TBR2) as measured by quantitative RT-PCR in 3-week-old organoids (Fig. 1I). Because reduced neural precursor proliferation may lead to cell cycle exit and differentiation, we next examined the level of neuronal markers DCX and CTIP2 (Fig. 1J-L). At 3 weeks, PTEN-OE organoids expressed increased levels of DCX and CTIP2 transcripts (Fig. 1J). Immuno-staining further confirmed that while few DCX- and CTIP2-positive neurons were present in control organoids, they were more abundant in PTENOE organoids (Fig. $1 \mathrm{~K}, \mathrm{~L}$ ). Therefore, our data indicates that increased dosage of PTEN reduces neural precursor proliferation, promotes premature neuronal differentiation, and results in the formation of significantly smaller brain organoids, mimicking the microcephalic condition seen in patients with 10q23.31 microduplication.

To investigate the molecular signaling that contributed to the cellular phenotypes, we next performed immunoblotting for activated (phosphorylated) AKT. Consistent with its function as a negative regulator of the PI3K-AKT pathway, we found that PTEN-OE organoids had reduced level of phospho-AKT (Fig. 1M, N and Additional file 1: Figure S1D). Because PTEN has functions dependent and independent of AKT activation, we investigated whether AKT inhibition in control organoids could recapitulate the PTEN-OE phenotype. We generated brain organoids from control hPSCs in the continuous presence of vehicle or $100 \mathrm{nM}$ MK-2206, a known AKT inhibitor, starting from day 1 of embryoid body formation. We have previously used MK-2206 to inhibit the aberrant AKT activation in PTEN knockout brain organoids and showed that it was effective in restoring normal organoid growth [10]. Here we found that MK-2206 treated organoids were significantly smaller than vehicle treated controls at 5-6weeks (Fig. 1O, P). Quantitative RT-PCR demonstrated that MK-2206 treatment led to decreased level of neural precursor markers (SOX2, TBR2) and increased presence of neuronal markers (DCX, CTIP2) (Fig. 1Q,
$\mathrm{R})$. The chronic treatment of MK-2206 mimicked the systemic overexpression of PTEN in patients and the in vitro organoid cultures. We further investigated the impact of short-term treatment of MK-2206 on hPSCs-derived neural precursors. We found that neural precursors cultured in the presence of growth factors (FGF2 and insulin) and $100 \mathrm{nM}$ MK-2206 for 7 days showed reduced AKT activity, as measured by the level of phospho-AKT (Additional file 1: Figure S2A, B) and phospho-S6, a downstream target of the AKT-mTOR pathway (Additional file 1: Figure S2A and C). While this treatment did not reduce SOX2 transcript level, it led to a significant decrease in TBR2, suggesting TBR2-positive intermediate progenitors may be more acutely vulnerable to AKT inhibition (Additional file 1: Figure S2D). Together, these findings suggest that increased dosage of PTEN leads to microcephaly in vitro by reducing AKT pathway activity.

In summary, our study provides functional evidence that increased dosage of PTEN contributes to impaired neurodevelopment in vitro. Given the known role of loss of PTEN in disorders including autism and macrocephaly, this new insight places PTEN amongst other dosage-sensitive causal genes (such as $M E C P 2$, $S H A N K 3, S C N 2 A, U B E 3 A$ ) for neurodevelopmental disorders. These findings are consistent with human genetics findings that aberrant activation and inhibition of the PI3K-AKT signaling pathway are both implicated in abnormal brain formation [11-14]. Future studies utilizing the hPSCs-derived brain organoid platform may provide additional insights into the disease etiology and therapeutic options for PTEN-related neurodevelopmental disorders.

The WIBR3 human embryonic stem cell line was approved for use by the Stem Cell Oversight Committee of the Canadian Institutes of Health Research, and the Research Ethics Board of the Hospital for Sick Children.

\section{Abbreviations}

PTEN: Phosphatase and tensin homolog; AKT: Ak strain transforming, also known as protein kinase B; PI3K: Phosphoinositide 3-kinase; hPSCs: Human pluripotent stem cells; OE: Overexpression; RT-PCR: Reverse transcription polymerase chain reaction.

\section{Supplementary Information}

The online version contains supplementary material available at https://doi. org/10.1186/s13041-021-00841-3.

Additional file 1: Figure S1. Analysis of PTEN-OE embryoid bodies and brain organoids. Figure S2. Short-term MK-2206 treatment of human neural precursors. Table S1. Antibody information. Table S2. Primer information.

Acknowledgements

The authors thank Jeff Lam, Erin Stout for technical support, and all members of the Li and Muffat labs for helpful discussions. 


\section{Authors' contributions}

$J M$ and $Y L$ designed the study. ND, WC, YL performed and analyzed the experiments. YL wrote the manuscript with input from all authors. All authors read and approved the final manuscript.

\section{Funding}

This research was part of the University of Toronto's Medicine by Design initiative, which received funding from the Canada First Research Excellence Fund. J.M. received funding from the Canada Research Chairs program. Work for this project was supported by grants from the Simons Foundation, the Ontario Institute for Regenerative Medicine, the Natural Sciences and Engineering Research Council of Canada, the Sharon Francis Institute for Regenerative Medicine, the Can-GARD, and the Hospital for Sick Children Restracomp scholarships

\section{Availability of data and materials}

All data generated or analyzed during this study are included in this article.

\section{Declarations}

\section{Ethics approval and consent to participate}

The WIBR3 human embryonic stem cell line was approved for use by theStem Cell Oversight Committee of the Canadian Institutes of Health Research,and the Research Ethics Board of the Hospital for Sick Children.

\section{Consent for publication}

Not applicable.

\section{Competing interests}

The authors declare no competing interests.

\section{Author details}

${ }^{1}$ Program in Developmental and Stem Cell Biology, The Hospital for Sick Children, 686 Bay Street, Toronto M5G 0A4, ON, Canada. ${ }^{2}$ Department of Molecular Genetics, University of Toronto, 1 King's College Circle, Toronto M5S 1A8, ON, Canada. ${ }^{3}$ Program in Genetics and Genome Biology, The Hospital for Sick Children, 686 Bay Street, Toronto M5G 0A4, ON, Canada. ${ }^{4}$ Program in Neurosciences and Mental Health, The Hospital for Sick Children, 686 Bay Street, Toronto M5G OA4, ON, Canada.

Received: 3 June 2021 Accepted: 19 August 2021

Published online: 30 August 2021

\section{References}

1. Winden KD, Ebrahimi-Fakhari D, Sahin M. Abnormal mTOR Activation in Autism. Annu Rev Neurosci. 2018:41:1-23.

2. Oliveira D, et al. 10q23.31 microduplication encompassing PTEN decreases mTOR signalling activity and is associated with autosomal dominant primary microcephaly. J Med Genet. 2019;56(8):543-7.

3. Omer Javed A, et al. Microcephaly modeling of kinetochore mutation reveals a brain-specific phenotype. Cell Rep. 2018;25(2):368-82. e365.

4. Lancaster $M A_{1}$ et al. Cerebral organoids model human brain development and microcephaly. Nature. 2013;501(7467):373-9.

5. Li R, et al. Recapitulating cortical development with organoid culture in vitro and modeling abnormal spindle-like (ASPM related primary) microcephaly disease. Protein Cell. 2017;8(11):823-33.

6. Esk C, et al. A human tissue screen identifies a regulator of ER secretion as a brain-size determinant. Science. 2020;370(6519):935-41.

7. Muffat J, et al. Human induced pluripotent stem cell-derived glial cells and neural progenitors display divergent responses to Zika and dengue infections. Proc Natl Acad Sci U S A. 2018;115(27):7117-22.

8. Qian X, et al. Brain-region-specific organoids using mini-bioreactors for modeling ZIKV exposure. Cell. 2016;165(5):1238-54.

9. Cugola FR, et al. The Brazilian Zika virus strain causes birth defects in experimental models. Nature. 2016;534(7606):267-71.

10. Li Y, et al. Induction of expansion and folding in human cerebral organoids. Cell stem cell. 2017;20(3):385-96 e383.

11. Boland $E$, et al. Mapping of deletion and translocation breakpoints in 1q44 implicates the serine/threonine kinase AKT3 in postnatal microcephaly and agenesis of the corpus callosum. Am J Hum Genet. 2007;81(2):292-303.

12. Lee $\mathrm{JH}_{\text {", }}$ et al. De novo somatic mutations in components of the PI3K-AKT3-mTOR pathway cause hemimegalencephaly. Nat Genet. 2012;44(8):941-5.

13. Riviere JB, et al. De novo germline and postzygotic mutations in AKT3, PIK3R2 and PIK3CA cause a spectrum of related megalencephaly syndromes. Nat Genet. 2012;44(8):934-40.

14. Poduri A, et al. Somatic activation of AKT3 causes hemispheric developmental brain malformations. Neuron. 2012;74(1):41-8.

\section{Publisher's Note}

Springer Nature remains neutral with regard to jurisdictional claims in published maps and institutional affiliations.
Ready to submit your research? Choose BMC and benefit from:

- fast, convenient online submission

- thorough peer review by experienced researchers in your field

- rapid publication on acceptance

- support for research data, including large and complex data types

- gold Open Access which fosters wider collaboration and increased citations

- maximum visibility for your research: over $100 \mathrm{M}$ website views per year

At BMC, research is always in progress.

Learn more biomedcentral.com/submissions 